\title{
Geometric Cues Influence Head Direction Cells Only Weakly in Nondisoriented Rats
}

\author{
Rebecca Knight, Robin Hayman, Lin Lin Ginzberg, and Kathryn Jeffery \\ Institute of Behavioural Neuroscience, Department of Cognitive, Perceptual and Brain Sciences, Division of Psychology and Language Sciences, University \\ College London, London WC1H 0AP, United Kingdom
}

The influential hypothesis that environmental geometry is critical for spatial orientation has been extensively tested behaviorally, and yet findings have been conflicting. Head direction (HD) cells, the neural correlate of the sense of direction, offer a window into the processes underlying directional orientation and may help clarify the issue. In the present study, HD cells were recorded as rats foraged in enclosures of varying geometry, with or without simultaneous manipulation of landmarks and self-motion cues (path integration). All geometric enclosures had single-order rotational symmetry and thus completely polarized the environment. They also had unique features, such as corners, which could, in principle, act as landmarks. Despite these strongly polarizing geometric cues, HD cells in nondisoriented rats never rotated with these shapes. In contrast, when a cue card (white or gray) was added to one wall, HD cells readily rotated with the enclosure. When path integration was disrupted by disorienting the rat, HD cells rotated with the enclosure even without the landmark. Collectively, these findings indicate that geometry exerts little or no influence on heading computations in nondisoriented rats, but it can do so in disoriented rats. We suggest that geometric processing is only a weak influence, providing a backup system for heading calculations and recruited only under conditions of disorientation.

\section{Introduction}

One of the most influential hypotheses concerning spatial orientation in animals is the geometric module hypothesis of Cheng (1986), which proposes that environmental geometry overrides features in orienting animals. This hypothesis has inspired much behavioral testing in many different species, but results have been mixed (for review, see Cheng and Newcombe, 2005), and the contribution of geometric processing to orientation remains incompletely understood (Cheng, 2008).

A possible complicating factor in behavioral studies is that determining an animal's heading calculation relies on measurement of a behavioral response such as goal localization, which is the end product of numerous processes over and above orientation per se, such as memory for the goal, use of environmental features as beacons rather than orienting cues, and navigational decision making. One way around this difficulty is to supplement behavioral observation with observation of head direction (HD) cells (Taube, 1995), which are neurons that encode the animals' directional heading in the

\footnotetext{
Received May 5, 2011; revised Aug. 17, 2011; accepted Aug. 22, 2011.

Author contributions: R.K. and K.J. designed research; R.K., R.H., and L.L.G. performed research; R.K. and R.H. analyzed data; R.K. and K.J. wrote the paper.

This work was supported by EU FP7 ("Spacebrain") and Wellcome Trust grants to K.J., and a Biotechnology and Biological Sciences Research Council studentship to R.K. and L.L.G. We thank Dina Lew for comments on the manuscript.

The authors declare no financial conflicts of interest.

This article is freely available online through the J Neurosci Open Choice option.

Correspondence should be addressed to Kathryn Jeffery, Institute of Behavioural Neuroscience, Department of Cognitive, Perceptual and Brain Sciences, Division of Psychology and Language Sciences, University College London, London WC1H OAP, UK. E-mail: k.jeffery@ucl.ac.uk.

DOI:10.1523/JNEUROSCI.2257-11.2011

Copyright $\odot 2011$ the authors $\quad 0270-6474 / 11 / 3115681-12 \$ 15.00 / 0$
}

horizontal plane and probably underlie the animal's sense of direction (Taube et al., 1996). Because the HD signal stands as proxy for the animal's directional sense, it provides a useful means of assessing the animal's calculated heading in the absence of behavioral confounds. We therefore used this signal to explore the use of geometric cues in heading determination.

We used three distinctly polarized geometric environments-a trapezoid, an isosceles triangle and a teardrop (Table 1) which varied in their geometric complexity and ambiguity and which were each situated within the center of a circular curtained arena. We show here that, surprisingly, despite the strong polarization of the environments, the HD cells in nondisoriented animals did not use the environments for orientation but instead remained aligned with the outside room. To rule out the possibility that room cues were unusually strong in our environment, we created fourth and fifth conditions by attaching either a gray or white cue card to the shortest wall of the triangle. As is typical (Taube et al., 1990b), HD cells rotated readily with the white, highly salient cue card and partially with the gray, less salient cue card. Finally, because behavioral experiments have typically used disoriented animals (Cheng, 1986; Wall et al., 2004; Pecchia and Vallortigara, 2010), and because orientation has also been shown to modulate the learning of geometric cues (Batty et al., 2009), we added a sixth condition where rats were disoriented between exposures to the triangle. Now HD cells oriented their firing using environment geometry.

Our results thus suggest that the influence of geometry on HD cells, which has been reported before (Clark et al., 2010), is evident only in highly disoriented rats. Under normal (nondisoriented) circumstances, it seems that environment geom- 
Table 1. A summary of the six conditions, along with the type of geometric cues available in each condition (not drawn to scale), the manipulation to path integration, and whether or not visual landmark information was available

\begin{tabular}{llll}
\hline Condition & Geometry & Path integration & Visual landmark \\
\hline Trapezoid & Orientated & Absent \\
Triangle & Orientated & Absent \\
Teardrop & Orientated & Absent \\
White cue card & Orientated & Present \\
Gray cue card & Orientated & Present \\
Disorientation & & \\
\hline
\end{tabular}

etry has little or no influence on heading computations in navigation.

\section{Materials and Methods \\ Subjects}

Sixteen adult male Lister Hooded rats (weighing between 273 and $465 \mathrm{~g}$ at the time of surgery) were housed individually [11:11 h light:dark, with $1 \mathrm{~h}(\times 2)$ simulated dawn/dusk] on a food-restricted diet sufficient to maintain $90 \%$ of free-feeding weight, with ad libitum access to water. All procedures were licensed by the UK Home Office subject to the restrictions and provisions contained in the Animals (Scientific Procedures) Act 1986

\section{Apparatus}

The experiment was performed in a circular arena $250 \mathrm{~cm}$ diameter, surrounded by thick black curtains. The arena was lit from directly above ( $\sim 250$ lux of light-approximately the same light level as an indoor office). Adjacent to the light was a video camera to record the location and orientation of the rat, and a small radio generating white noise to overshadow external uncontrolled auditory cues. Recordings in this arena took place inside one of three geometric enclosures, which were constructed from hardboard, painted black, and had no floor or ceiling (Fig. 1A). The enclosures comprised a trapezoid, an isosceles triangle, and a teardrop, chosen for the reasons outlined below.

Trapezoid. The trapezoid was regular (mirror-symmetric). The parallel walls were 60 (short wall) and 120 (long wall) cm long; the angled walls were both $70 \mathrm{~cm}$ long. All walls were $60 \mathrm{~cm}$ high. This enclosure had two pairs of congruent angles of $65^{\circ}$ and $115^{\circ}$, respectively, so geometric information emanating from the corners would be ambiguous. Thus, any ability of the directional system to use geometry to orient unambiguously would require either an ability to discriminate wall length (since there was a unique shortest wall and a unique longest wall) or an ability to integrate information from more than one corner, or from a corner and a wall, simultaneously.

Isosceles triangle. The triangle enclosure was designed as a response to the finding that HD cells did not rotate with the trapezoid. The triangle had two equal long walls of $122 \mathrm{~cm}$ and a single short wall of $60 \mathrm{~cm}$, making a single unique angle of $30^{\circ}$ and two congruent angles of $75^{\circ}$ flanking the base of the triangle. The height of the walls was $60 \mathrm{~cm}$. We considered that the increased aspect ratio of the triangle, together with the presence of one very obvious, narrow corner, would enhance the likelihood that HD cells would use this feature of the geometry to orient, if orientation by geometric cues occurs at all.

Teardrop. The teardrop was introduced following the surprising finding that HD cells did not rotate with the isosceles triangle either. We hypothesized that perhaps the cells were unable to use wall length as a cue, and that the three angles of the triangle might not have been sufficiently discriminable to aid orientation. Thus, the teardrop possessed only a single corner with an angle similar to that in the triangle $\left(30^{\circ}\right)$. If HD cells can use corners as a geometric cue, then the teardrop should provide unambiguous polarizing information to them.

Triangle plus gray or white cue card. The failure of HD cells to rotate their firing with the above enclosures might have been due to their small size-perhaps HD cells cannot use information of such close proximity. Alternatively, perhaps enclosure size is irrelevant but the cells prefer visual information, which was impoverished in the geometric enclosures due to the low contrast provided by corners etc. In these manipulations, therefore, a cue card of high contrast (white) or low contrast (gray) was introduced to the isosceles triangle. As we will show, cells rotated readily with the white cue card and somewhat with the gray card.

For each session, one of these enclosures was placed in the center of the arena, directly onto two black plastic sheets that could be wiped and rotated between trials to scramble olfactory cues. The orientation of the arena on the first trial of each day was pseudorandomized so that the orientation was never the same for consecutive days. To maximize the chance of the rats relying on the geometric properties of the shape for orientation, no polarizing extramaze cues were placed anywhere in the arena. For cue card trials, either a white or a gray A3 card was taped to the inside of the isosceles triangle's shortest wall (Fig. 1A).

\section{Surgery and electrodes}

All rats were implanted at the start of the experiment with moveable microelectrodes. Four tetrodes were constructed from four interwound, $25-\mu \mathrm{m}$-diameter platinum-iridium wire (California Fine Wire). The tetrodes were held in a microdrive assembly (Axona) that allowed them to be lowered or raised, with one full turn of the screw equal to an increment of $200 \mu \mathrm{m}$ dorsoventrally. During surgery, the animals were anesthetized with isoflurane and oxygen $(3 \mathrm{l} / \mathrm{min})$. Animals were placed on a stereotaxic frame, with lambda and bregma in the horizontal plane. Microdrives were fixed to the skull with six $1.6 \mathrm{~mm}$ jewelers' screws (Precision Technology Supplies) and dental cement. One of the screws was soldered to a ground wire to enable the animal to be electronically grounded. The electrodes were lowered into the brain in areas known to contain HD cells: the postsubiculum ( $\mathrm{PoS})$, the anterodorsal thalamic nucleus $(\mathrm{ADN})$, the retrosplenial cortex (RSP), or the lateral mammillary nuclei (LMN). Four HD cells were also recorded from two animals that were implanted for a separate experiment using coordinates for the CA1 region of the hippocampus, which has been shown to contain HD cells (Leutgeb et al., 2000). Once the electrodes were implanted, a metallic sleeve was pulled down over the remaining exposed wires. Postsurgery, the animals were monitored periodically until they awoke. All animals were given at least 1 week to recover following the surgery.

\section{Screening procedures}

Recording commenced 1 week after surgery. Recording was done using multichannel recording equipment (DacqUSB, Axona). The rats were connected to the recording device via lightweight wires and a socket attached to the microdrive plug. The potentials recorded on each of the 16 electrodes of the four tetrodes were passed through AC-coupled unity gain operational amplifiers mounted on the rat's head and fed to the recording system. The signal was amplified $(\sim 20,000 \times)$, bandpass filtered $(300 \mathrm{~Hz}-7 \mathrm{kHz})$, collected, and stored on a computer. Each of the four wires of one tetrode was recorded differentially with respect to the wires of other tetrodes. Screening for HD cells took place in a room separate from the actual experimental room to minimize the learning of extraneous cues in the recording environment by the rats. During screening, rats were encouraged to completely sample the floor surface by for- 
A

\section{Trapezoid Condition}

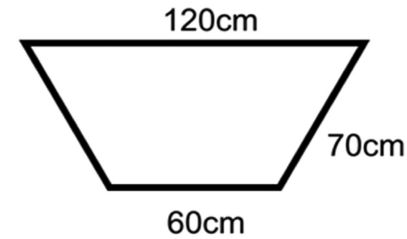

Triangle Condition

B

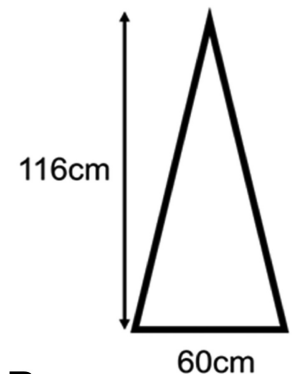

\section{Protocol for one triangle session}

$90^{\circ} \mathrm{CW}$

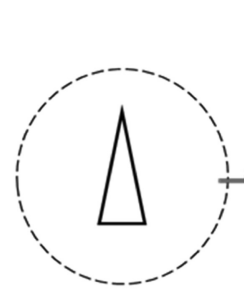

Trial 1

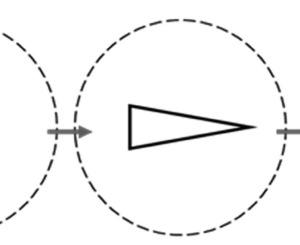

Trial 2 $180^{\circ}$

$90^{\circ} \mathrm{CCW}$

$180^{\circ}$
Teardrop Condition

$113 \mathrm{~cm}$

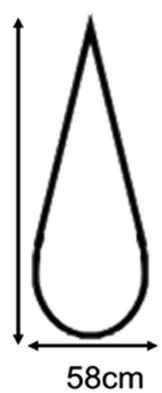

Cue Card Conditions

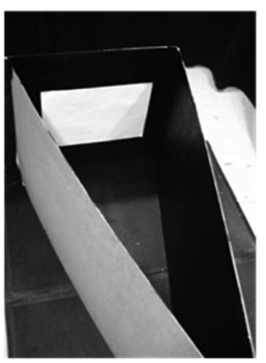

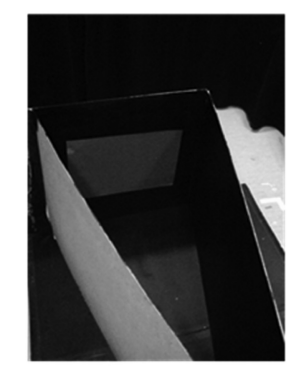

Figure 1. A, The dimensions of the environments used for each condition, along with photos of the $A 3$ cue cards attached to the shortest wall of the isosceles triangle. All environments were constructed using hardboard and painted matte black. The height of all the environments measured $60 \mathrm{~cm}$. The teardrop shape environment was constructed from a flexible piece of hardboard inserted inside the isosceles triangle. $\boldsymbol{B}$, An example of a protocol used in one session in the triangle condition. The outline of a triangular environment indicates how the environments were rotated between trials inside the curtained environment. Each session consisted of two $180^{\circ}$ and two $90^{\circ}$ [ clockwise (CW) and counterclockwise (CCW)] rotations. To achieve this, $90^{\circ}$ and $180^{\circ}$ rotations had to be interleaved, which meant that four sequences of rotations were possible. These four sequences were pseudorandomized.

aging for Coco Pops (Kellogg's). A directional head-stage with two LED arrays, one large and one small, aligned opposite each other at a separation of $10 \mathrm{~cm}$, was used along with a video camera attached to the ceiling to track the rat's location and head direction. If no HD cell activity was present, the electrodes were lowered by $\sim 50 \mu \mathrm{m}$.

\section{Recording procedures}

When an HD cell was found, the rat was taken into the experimental room in an opaque holding box and placed on a raised platform box outside of the curtained enclosure to acclimatize to the room for $5 \mathrm{~min}$. The rat was connected to the recording device while outside of the curtained enclosure, then returned to the holding box, which was carried into the enclosure through one of three joins in the curtains and placed in a pseudorandom location on the floor within the curtained area. After 1 min, the rat was taken out of the holding box, carried $\sim 1 \mathrm{~m}$, and placed in the center of the geometric enclosure, using a different point of entry for each trial. The lightweight wires were attached by clips to two pieces of elastic string suspended from the ceiling and the recording session, which comprised five individual trials, commenced. Food was thrown into the environment to encourage rats to sample the whole space. During this time, the empty holding box was moved to a new location in the curtained enclosure to prevent it from becoming a stable directional cue. Each trial lasted for $240 \mathrm{~s}$, after which the wires were unclipped from the string and the rat was removed from the environment. The rat was placed back in the holding box, which now occupied a new location in the curtained enclosure. The rat remained in the holding box while the environments were rotated.

While the rat was in the holding box, the two floor cards were flipped, shuffled, and cleaned with water. The geometric enclosure was also flipped upside down and rotated by either $90^{\circ}$ (clockwise or counterclockwise) or $180^{\circ}$. Rotations were pseudorandom, so that each recording session contained one $90^{\circ}$ clockwise rotation, one $90^{\circ}$ counterclockwise rotation, and two $180^{\circ}$ rotations. Thus, the shape was aligned with each of the compass axes once (north, south, east, west), except for the last trial, when the shape's orientation was the same as that in the first trial (Fig. $1 B$ ).

Before the rat was placed back in the newly rotated environment, it either did or did not undergo a disorientation treatment, which disrupts path integration. For five of the six conditions, the rat was not disoriented, because previous findings have shown that this can prevent a rat from forming a stable relationship between its internal sense of direction and the environment (Knierim et al., 1995). However, in the remaining condition, the holding box was manually spun by the experimenter (approximately one rotation per second) while they walked around the curtained enclosure for $\sim 1 \mathrm{~min}$.

Then, for all six condition types, the rat was taken out of the holding box and placed back in the center of the shape for a further $240 \mathrm{~s}$ trial. The protocol was repeated until the rat had been recorded in the five trials, at which point the session was complete and the rat was taken out of the curtained enclosure in the holding box, disconnected from the recording cable, and returned to the home cage. The presentation order for each condition was pseudorandomized, ensuring that each of the six conditions was the first condition for at least one rat and the last condition for at least one rat (Table 2).

\section{Data analysis}

Cluster-cutting software (Tint; Axona) was used to analyze the data offline. The peak-to-peak amplitude for one electrode was plotted against each of the other electrodes in the tetrode, creating clusters of spikes that allowed cells to be isolated by hand. All cells with a peak firing rate of $<1.0 \mathrm{~Hz}$ across the session were excluded from further analysis. For the remaining data, polar plots (firing rate as a funciton of head direction) were created for each cell's directional activity using a smoothing kernel of $5^{\circ}$. To calculate each cell's mean firing direction, peak firing rate, and directional firing range for each trial, the animal's head direction was divided into $606^{\circ}$ bins and smoothed in Matlab (Mathworks) using a smoothing kernel of $5^{\circ}$. To calculate the directional firing range, a trian- 
Table 2. The presentation order of conditions for each rat

\begin{tabular}{|c|c|c|c|c|c|}
\hline Rat & Location & 1st condition & 2nd condition & 3rd condition & 4th condition \\
\hline R318 & CA1 (Total 2 cells) & Triangle × 2 (2 ensembles) & & & \\
\hline R321 & PoS (Total 9 cells) & Trapezoid ×4 (1 ensemble) & Triangle (1 ensemble) & & \\
\hline R330 & CA1 (Total 1 cells) & Trapezoid (1 ensemble) & & & \\
\hline R333 & ADN (Total 1 cell) & Trapezoid (1 ensemble) & Triangle (1 ensemble) & & \\
\hline R344 & PoS (Total 8 cells) & Triangle $\times 5$ ( 1 ensemble) & Disorientation (1 ensemble) & Trapezoid (1 ensemble) & \\
\hline R371 & PoS (Total 5 cells) & White card (1 ensemble) & Triangle (1 ensemble) & Trapezoid (1 ensemble) & \\
\hline R388 & RSP (Total 1 cell) & White card (1 ensemble) & & & \\
\hline R404 & RSP (Total 2 cells) & Gray card (1 ensemble) & Teardrop (1 ensemble) & & \\
\hline R409 & ADN (Total 3 cells) & White card (1 ensemble) & Teardrop (2 ensembles) & Triangle (1 ensemble) & Grey card (1 ensemble) \\
\hline R410 & ADN (Total 1 cell) & Gray card (1 ensemble) & & & \\
\hline R420 & ADN (Total 1 cells) & Gray card $\times 2$ ( 2 ensembles) & White card $\times 2$ (1 ensemble) & Gray card (1 ensemble) & Disorientation $\times 2$ ( 1 ensemble) \\
\hline R1693 & LMN (Total 1 cell) & Triangle (1 ensemble) & & & \\
\hline R1696 & RSP (Total 4 cells) & Gray card (1 ensemble) & Teardrop $\times 2$ ( 2 ensembles) & & \\
\hline R1697 & RSP (Total 2 cells) & White card (1 ensemble) & Teardrop (1 ensemble) & & \\
\hline R1704 & RSP (Total 3 cells) & Teardrop (1 ensemble) & White card (1 ensemble) & & \\
\hline R433 & PoS (Total 2 cells) & Disorientation $\times 2$ ( 2 ensembles) & & & \\
\hline
\end{tabular}

All conditions were presented first for at least one rat and presented last for at least one rat in order to counterbalance any order effects. The table also presents the brain area where each rat was implanted, the total number of $\mathrm{HD}$ cells recorded in each rat, and the total number of ensembles recorded in each condition for each rat.

gle was fitted to a firing rate versus HD plot. Using Taube et al.'s (1990a) criteria, left and right maximum and left and right minimum data points were selected to form a triangular shape. A linear set of values was then extrapolated for the right and left legs. The base of the triangle (where the two legs intersected the $x$-axis) was then taken as the directional firing range. To assess the degree to which the data deviated from the circular mean, mean vector lengths were calculated on unsmoothed data. Mean vector lengths $(r)$ measured the circular spread of data with values ranging from 0 to 1 . Mean vector length $r$ values that are closer to 1 indicate less variance in the dataset. HD cells that had an $r$ value of $<0.3$ within a given session were excluded (van der Meer et al., 2010). Figure $2 A$ shows an example of one HD cell from the PoS that had an $r$ value of 0.66 pre- $180^{\circ}$ rotation. Figure $2, B$ and $C$, shows an example of one HD cell from the ADN with an $r$ value of 0.71 and from an RSP cell with an $r$ value of 0.88 , respectively.

Because HD cells from a given animal always behave coherently (Taube et al., 1990b) and cannot be treated as independent data, from this point onwards in the analysis any subsequent calculations were performed using the circular mean values of cells that had been recorded simultaneously, i.e., as an ensemble (ranging from 1 to 7 cells). All analysis was done using the CircStat Matlab toolbox (Berens, 2009). To analyze the rotation of HD cells from one trial to the next, the circular mean direction of cells in the first trial was subtracted from the circular mean direction of cells in the second trial (Fig. 2) to provide a measure of how far the ensemble had rotated. This was repeated for each subsequent trial of the session so that each session produced four rotation estimates. These mean rotations were then subtracted from the predicted angle of shift, based on how much the environment had rotated, to produce absolute deviations from expected rotation. The mean vector length of absolute deviations for each condition was calculated. The Rayleigh test (Berens, 2009) was then used to determine whether these absolute mean deviations clustered around a particular direction. Circular one- and two-sample $t$ tests (Berens, 2009) were used to compare absolute mean deviation values against zero (the predicted deviation given perfect enclosure-following) and to compare absolute mean deviation values between conditions, respectively. A mean absolute deviation, which was not significantly different from zero, indicates that the HD cells followed the rotation of the shaped enclosure.

\section{Histological analysis}

At the end of testing, the rats were deeply anesthetized with isoflurane before they were injected with sodium pentobarbital. They were then transcardially perfused using saline followed by paraformaldehyde (4\%). The brains were removed and stored in paraformaldehyde (4\%) for at least 1 week before sectioning. The brains were sliced at $40 \mu \mathrm{m}$ on a freezing microtome. The sections were then mounted and stained with cresyl violet (Fig. 3).

\section{Results}

The environments used in the recordings are shown in Figure $1 \mathrm{~A}$, and a schematic protocol of one recording session (a sequence of five trials) is shown in Figure $1 \mathrm{~B}$. A total of 56 individual HD cells were recorded from 16 rats during 47 sessions. Simultaneously recorded cells were grouped together as single ensembles, ranging from one to seven cells, of which there were 22 in total. Below, we first present descriptive data from the individual neurons, and then present the data from the ensembles, which we take as proxy for the rat's internal direction sense.

Fifty-six cells met the inclusion criteria, with a peak firing rate of $>1.0 \mathrm{~Hz}$ and a mean vector length $(r)$ of $>0.3$ (van der Meer et al., 2010). Of these 56 cells, histological analysis (Fig. 3) showed that 24 were recorded in the PoS, 16 in the ADN, 12 in the RSP, and one in the LMN (Table 2). The three remaining cells were recorded from rats implanted using CA1 coordinates [where HD cells are sometimes found (Leutgeb et al., 2000)], although this could not be confirmed with histology. Overall, these $56 \mathrm{HD}$ cells had an average peak firing rate of $21.6 \mathrm{~Hz}$ and an average directional firing range of $109^{\circ}$. Circular statistical analysis determined the mean vector length, or $r$ value, which is a measure of the spread of the data ranging from 1 (perfectly concentrated) to 0 (randomly distributed around all directions). The mean $r$ value for the complete HD cell dataset was 0.5 .

Comparisons were made between these brain areas to determine whether there were significant differences in peak firing rate, directional firing range, and $r$ values of the cells. As only one cell was recorded from the LMN and the three cells implanted with CA1 coordinates could not be confirmed, these areas were excluded from comparisons. Of the three parameters, peak firing rate $\left(F_{(2,49)}=6.04, p<0.05\right)$ showed overall significant differences. Post hoc tests (with Bonferroni corrections) at an alpha level of 0.05 found that cells in the ADN had significantly higher peak firing rates compared with the PoS (upper CI $=39.87$, lower $\mathrm{CI}=5.76$ ). Figure 2 shows prerotation and postrotation tuning curves of one cell recorded in the ADN, one cell in the PoS, and one cell in the RSP.

During each session, rats were exposed to one of the environments over five trials. Between trials, the environments were rotated by $90^{\circ}$ clockwise, $90^{\circ}$ counterclockwise, or $180^{\circ}$, during which time we took care not to disorient the rats (except for condition 6) (Knierim et al., 1995). There were no overall signif- 

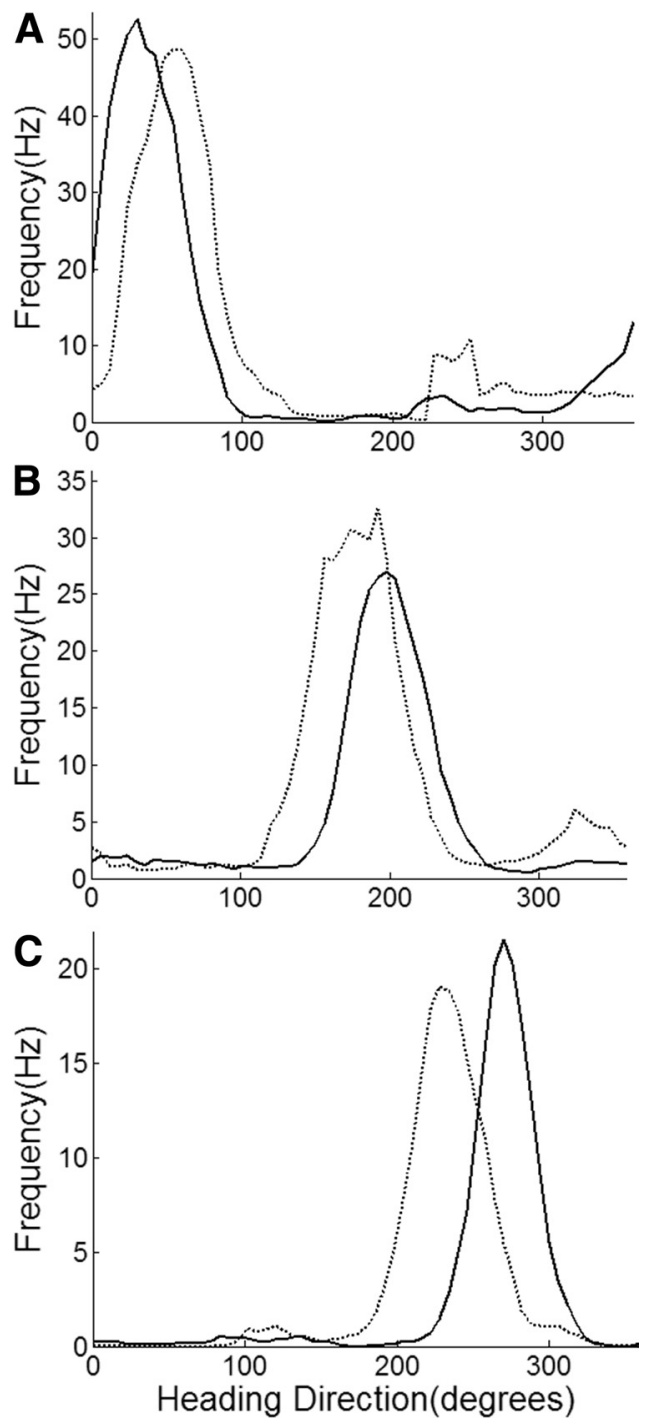

Figure 2. $\quad \boldsymbol{A}-\boldsymbol{C}$, Examples of single-cell firing profiles from the $\operatorname{PoS}(\boldsymbol{A}), \operatorname{ADN}(\boldsymbol{B})$, and $\operatorname{RSP}(\boldsymbol{C})$. $A$, PoS cell during a $180^{\circ}$ rotation in a trapezoid trial. The plot shows the tuning curve of an HD cell prerotation (solid line) and postrotation (dotted line). The HD cell for the prerotation session had an $r$ value of 0.66 . For each rotation, the mean shift was calculated by taking the mean of the prerotation tuning curve and subtracting it from the mean of the postrotation tuning curve. For this example, the mean shift was $22.16^{\circ}$. B, ADN cell during a $90^{\circ}$ rotation in a teardrop trial, where the cell had a prerotation $r$ value of 0.71 . C, RSP cell during a $90^{\circ}$ rotation in a teardrop trial, where the cell had a prerotation $r$ value of 0.88 . Statistics showed that HD cells in the ADN had significantly higher peak firing rates compared with POS HD cells. There were no significant differences in directional firing ranges and $r$ values of $\mathrm{HD}$ cells in the POS, ADN, and RSP.

icant differences in peak firing rate for pre- and post- $90^{\circ}$ rotations $\left(21.6\right.$ and $22.02 \mathrm{~Hz}$, respectively; $t_{(1,204)}=-0.468$, NS) or pre- and post- $180^{\circ}$ rotations $(21.77$ and $22.12 \mathrm{~Hz}$, respectively; $t_{(1,204)}=-0.563$, NS). There were also no overall significant differences in directional firing range for pre- and post- $90^{\circ}$ rotations $\left(108.6^{\circ}\right.$ and $108.5^{\circ}$, respectively; $\left.t_{(1,204)}=0.059, \mathrm{NS}\right)$ or pre- and post $-180^{\circ}$ rotations $\left(109.1^{\circ}\right.$ and $108.7^{\circ}$, respectively; $t_{(1,204)}=0.144$, NS).

Henceforth, the analyses detailed below consider the data at ensemble level rather than single-cell level. No differences in ensemble behavior between clockwise and counterclockwise rotations were noted. Thus, in the analyses to follow, rotations of $90^{\circ}$ clockwise and $90^{\circ}$ counterclockwise were equated by a normalization process in which the counterclockwise data were reflected in the $0-180^{\circ}$ axis to map them onto the clockwise data.

\section{Condition 1: Trapezoid}

An example of a session in the trapezoid is shown in Figure $4 \mathrm{~A}$. Note that the firing direction of the cell did not follow the enclosure except on the first trial; thereafter, it resumed and maintained a stable orientation with respect to the room. A stable orientation could be due to a stable path integration cue or uncontrolled extramaze cues. Indeed, we cannot entirely rule out the possibility that rats were using extramaze cues during some trials, or more likely a combination of both extramaze cues and path integration. Efforts were made to minimize the influence of any extramaze cues in all conditions. For example, white noise was played and a black curtain surrounded the environment. However, there may have been odor or auditory cues evident to the rat but not the experimenter.

A total of $16 \mathrm{HD}$ cells, comprising five ensembles, were recorded in the trapezoid from five rats over eight sessions. The data from the collection of sessions are shown in Figure $5 A$. The subsequent statistical analyses looked at how much the cells' firing directions remained locked onto the room cues versus rotated with the enclosure (the deviation-from-expected measure) and whether the resulting distribution was randomly scattered or clustered around a particular direction. We present the data from the $90^{\circ}$ rotations first, followed by the $180^{\circ}$ rotations.

Overall, for all ensembles during a $90^{\circ}$ trapezoid rotation, the mean shift in firing direction with respect to the room beyond the curtains was $-1^{\circ}$, with a resultant vector length of 0.89 (Fig. $5 A$ ). A Rayleigh test showed that these data significantly clustered around the $-1^{\circ}$ shift $(r=7.16, p<0.05)$ and were not significantly different from zero (upper $\mathrm{CI}=25.78$, lower $\mathrm{CI}=$ -28.07 ), indicating a strong propensity for the cells to retain their orientation with respect to the outside room. Correspondingly, we found a final deviation-from-expected value of $91^{\circ}$ ( $\pm 26.33 \mathrm{SD}$ ), which was significantly different from zero (upper $\mathrm{CI}=118.0$, lower $\mathrm{CI}=64.2$ ). Together, these results indicate that the rotations of the firing directions of the HD cells were significantly different from rotations of the trapezoid environment and were not different from the direction predicted by the room.

For all ensembles during a $180^{\circ}$ trapezoid rotation, the mean shift in firing direction was $-14^{\circ}$, with a resultant vector length of 0.9 (Fig. 5A). A Rayleigh test showed that these data significantly clustered around the $-14^{\circ}$ value $(r=7.21, p<0.01)$ and were not significantly different from zero (upper CI $=8.59$, lower $\mathrm{CI}=-36.1$ ), indicating a strong propensity for the cells to remain oriented to the room. Correspondingly, we found a final deviation-from-expected value of $-166^{\circ}$ ( $\left.\pm 25.45 \mathrm{SD}\right)$, which was significantly different from zero (upper CI $=-143.8$, lower $\mathrm{CI}=-188.5$ )

Thus, the trapezoid, despite its polarized geometry, failed to gain control of HD cells, which instead appeared to lock onto uncontrolled cues. The next manipulation aimed to test this by introducing an environment (an isosceles triangle) that was even more polarized, with a larger difference between the short wall and long walls, and a unique acute angle.

\section{Condition 2: Triangle}

A total of $15 \mathrm{HD}$ cells (eight ensembles) were recorded in the triangle from seven rats over 12 sessions. An example of a session in the triangle is shown in Figure $4 B$. In this example, which was representative, the firing direction of the cell did not rotate with the enclosure.

Overall, for all ensembles during a $90^{\circ}$ triangle rotation, the mean shift in firing direction was $-13^{\circ}$, with a resultant vector length of 0.52 (Fig. $5 B$ ). A Rayleigh test showed that these data 

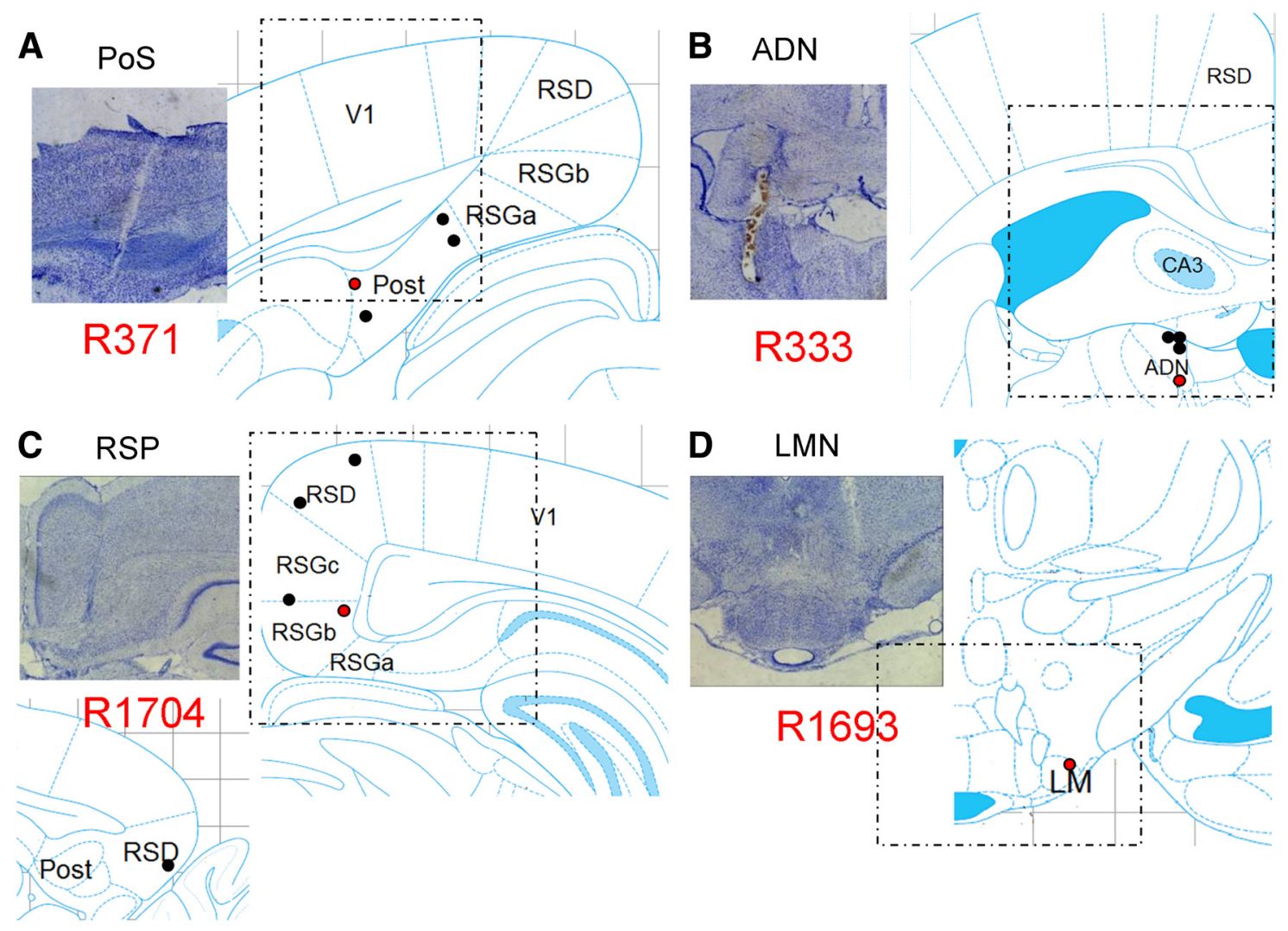

Figure 3. $\quad \boldsymbol{A}-\boldsymbol{D}$, Histology from postsubiculum (Post; $\boldsymbol{A}), \operatorname{ADN}(\boldsymbol{B})$, retrosplenial cortex (dysgranular, RSD; granular a, RSGa; granular b, RSGb; granular c, RSGc; $\boldsymbol{C}$, and lateral mammillary nucleus $(L M ; D)$. An example slice is shown for each brain region, along with the corresponding red dot on the template (Paxinos and Watson, 2004). Each black dot represents the recording site for the remaining rats implanted in that region. The histology for the two rats implanted in the CA1 region of the hippocampus could not be recovered. They were, however, implanted using the following coordinates: anterior/posterior: - 3.5; medial/lateral: 2.0; dorsal/ventral: - 1.5. Place cells were also found in this region. R371, R333, R1704, and R1693 are the identifiers of the rats whose data is shown here.

significantly clustered around the $13^{\circ} \operatorname{shift}(r=6.78, p<0.05)$ and were not significantly different from zero (upper $\mathrm{CI}=57.87$, lower $\mathrm{CI}=-32.66$ ), indicating, as with the trapezoid, a strong propensity for the cells to remain oriented to the room. Correspondingly, we found a final deviation-from-expected value of $77^{\circ}( \pm 56.0 \mathrm{SD})$, which was significantly different from zero (upper $\mathrm{CI}=122.61$, lower $\mathrm{CI}=31.51$ ). Together, these results indicate that the rotations of the firing directions of the HD cells were significantly different from rotations of the triangle environment and were not different from the direction predicted by the room.

Similarly, for all ensembles during a $180^{\circ}$ triangle rotation, the mean shift in firing direction was $2^{\circ}$, with a resultant vector length of 0.75 (Fig. $5 B$ ). A Rayleigh test showed that these data significantly clustered around the $2^{\circ}$ value $(r=9.72, p<0.01)$ and were not significantly different from zero (upper $\mathrm{CI}=29.22$, lower $\mathrm{CI}=-25.21$ ), indicating a strong propensity for the cells to remain oriented to the room. Correspondingly, we found a final deviation-from-expected value of $178^{\circ}( \pm 40.69 \mathrm{SD})$, which was significantly different from zero (upper $\mathrm{CI}=205.12$, lower $\mathrm{CI}=$ 151.26).

One possible reason for the failure of cells to lock onto the geometry of the triangle is that from the perspective of the rat, the corners look similar and the wall lengths are hard to discriminate. This seems surprising, but nevertheless, we attempted to make the environment less rotationally ambiguous by reducing the number of corners to one (the teardrop enclosure).

\section{Condition 3: Teardrop}

A total of $12 \mathrm{HD}$ cells (seven ensembles) were recorded in the teardrop from five rats over seven sessions. An example of a session in the teardrop is shown in Figure 4C. Note that, as in the trapezoid and the triangle, the firing direction of the cell did not rotate with the enclosure.

Overall, for all ensembles during a $90^{\circ}$ teardrop rotation, the mean shift in firing direction was $-23^{\circ}$, with a resultant vector length of 0.77 (Fig. 5C). A Rayleigh test showed that these data significantly clustered around the $23^{\circ}$ shift $(r=5.41, p<0.01)$ and were not significantly different from zero (upper $\mathrm{CI}=60.73$, lower $\mathrm{CI}=-14.9$ ), indicating a strong propensity for the cells to remain oriented to the room. Correspondingly, we found a final deviation-from-expected value of $67^{\circ}( \pm 38.62 \mathrm{SD})$, which was significantly different from zero (upper $\mathrm{CI}=104.85$, lower $\mathrm{CI}=$ 29.79). Together, these results indicate that the rotations of the firing directions of the HD cells were significantly different from rotations of the teardrop environment and were not different from the direction predicted by the room.

Similarly, for all ensembles during a $180^{\circ}$ teardrop rotation, the mean shift in firing direction was $12^{\circ}$, with a resultant vector length of 0.9 (Fig. 5C). A Rayleigh test showed that these data significantly clustered around the $12^{\circ}$ value $(r=6.29, p<0.01)$ and were not significantly different from zero (upper $\mathrm{CI}=41.25$, lower $\mathrm{CI}=-17.19$ ), indicating a strong propensity for the cells to remain oriented to the room. Correspondingly, we found a final deviation-from-expected value of $168^{\circ}( \pm 25.75 \mathrm{SD})$, which 
A

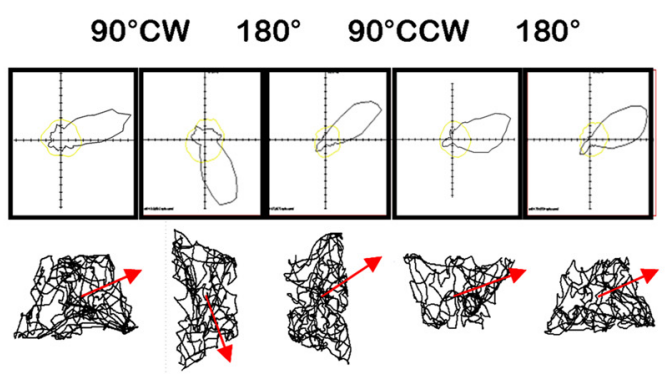

$\begin{array}{lllll}\text { Trial } 1 & \text { Trial } 2 & \text { Trial } 3 & \text { Trial } 4 & \text { Trial } 5\end{array}$

C

Teardrop

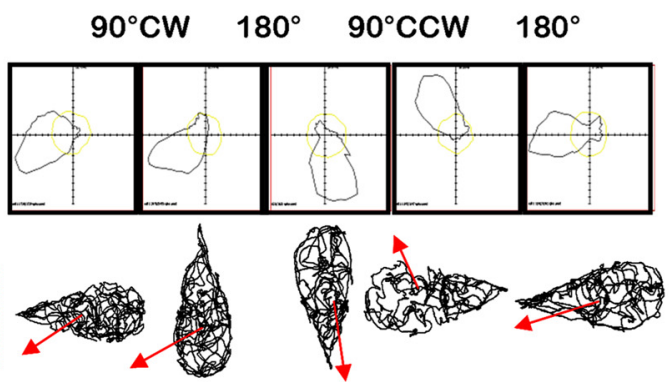

$\begin{array}{lllll}\text { Trial } 1 & \text { Trial } 2 & \text { Trial } 3 & \text { Trial } 4 & \text { Trial } 5\end{array}$

E

Gray Cue Card

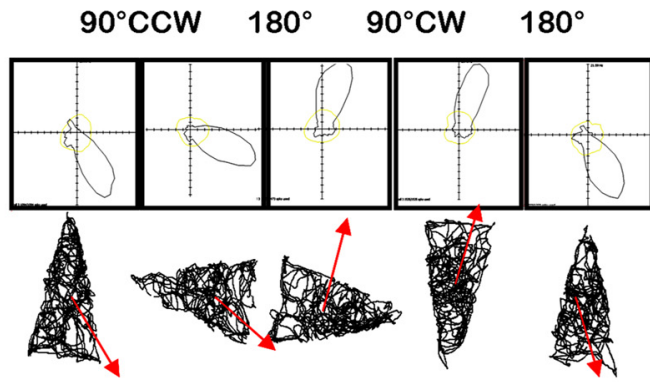

$\begin{array}{lllll}\text { Trial } 1 & \text { Trial } 2 & \text { Trial } 3 & \text { Trial } 4 & \text { Trial } 5\end{array}$
B Triangle

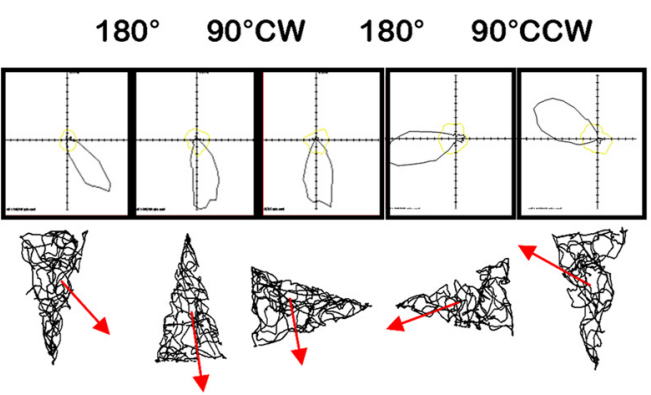

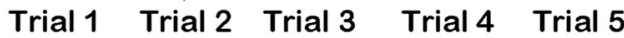

D White Cue Card

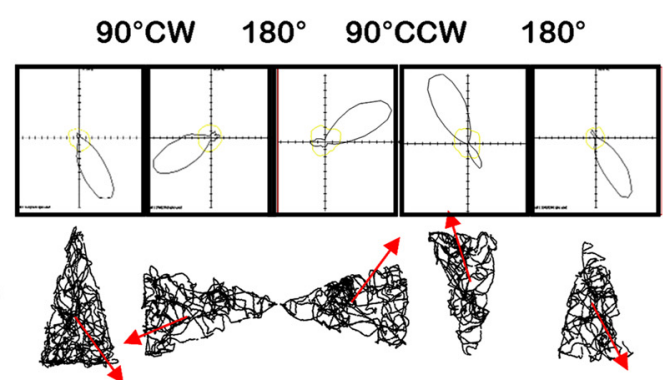

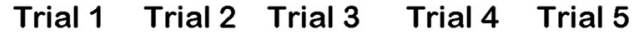

F Disorientation

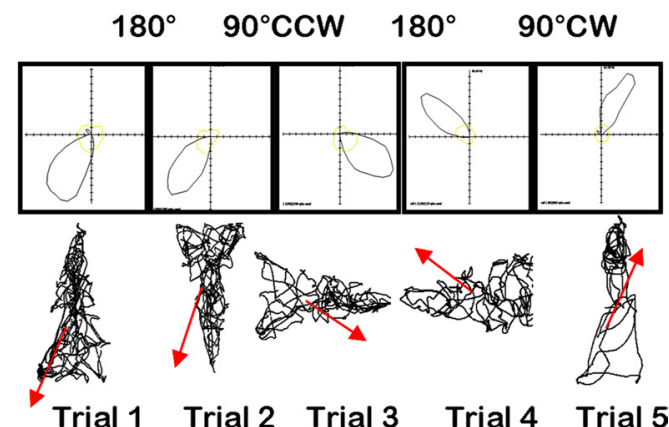

Figure 4. $\quad \boldsymbol{A}-\boldsymbol{F}$, An example of the results produced during one trapezoid session $(\boldsymbol{A})$, one triangle session $(\boldsymbol{B})$, one teardrop session ( $\boldsymbol{C}$, one white cue card session $(\boldsymbol{D})$, one gray cue card session $(\boldsymbol{E})$, and one disorientation session $(\boldsymbol{F})$. The polar plots indicate the firing direction of one cell across the five trials. The corresponding paths that the rat took for each trial are shown along with a superimposed red line to illustrate how the rotation of the HD cell corresponded to the rotation of the environment. CW, Clockwise; CCW, counterclockwise.

was significantly different from zero (upper CI $=197.1$, lower $\mathrm{CI}=139.23)$.

The failure of HD cells to rotate their firing even in this unambiguously polarized teardrop condition suggests that perhaps HD cells are incapable of following geometric cues. However, it could be argued that, in a situation where cue conflict is very strong (geometry conflicting with uncontrolled auditory and olfactory cues, as well as the rat's internal sense of direction), HD cells may be unable to reliably use any local environmental cue. To test this possibility, a landmark (a white cue card) was attached to the shortest wall of the isosceles triangle. As we show below, the white cue card readily gained control of the cells.

Condition 4: Triangle plus white cue card

A total of $15 \mathrm{HD}$ cells (six ensembles) were recorded from six rats during seven sessions when a white A3 cue card was attached to the shortest wall of the triangle. An example of a trial in the white cue card condition is shown in Figure 4D. Note that, unlike the previous examples, in this representative example, the firing direction of the cell reliably followed the triangle plus white card.

Overall, for all ensembles during a $90^{\circ}$ triangle plus white card rotation, the mean shift in firing direction was $85^{\circ}$, with a resultant vector length of 0.99 (Fig. 5D). A Rayleigh test showed that these data significantly clustered around the $85^{\circ}$ shift $(r=$ 7.78, $p<0.01$ ) and were significantly different from zero (upper $\mathrm{CI}=92.82$, lower $\mathrm{CI}=77.35$ ), indicating that the cells did not remain oriented to the room. Correspondingly, we found a final deviation-from-expected value of $5^{\circ}( \pm 9.62 \mathrm{SD})$, which was not significantly different from zero (upper CI = 12.61, lower CI $=-2.86$ ), indicating that the cells had followed the white cue card.

Similarly, for all ensembles during a $180^{\circ}$ triangle plus white card rotation, the mean shift in firing direction was $173^{\circ}$, with a resultant vector length of 0.81 (Fig. 5D). A Rayleigh test showed 
that these data significantly clustered around the $173^{\circ}$ value $(r=6.5, p<0.01)$ and were significantly different from zero (upper $\mathrm{CI}=204.55$, lower $\mathrm{CI}=141.52$ ), indicating that the cells did not lock onto the room cues. Correspondingly, we found a final deviation-from-expected value of $7^{\circ}( \pm 35.0 \mathrm{SD})$, which was not significantly different from zero (upper $\mathrm{CI}=$ 38.96, lower CI $=-24.64)$. Together, these results show that the rotations of the firing directions of the HD cells were not significantly different from rotations of the triangle plus white card environment, indicating that the firing directions remained generally aligned to the cue card.

We wondered whether visual saliency might explain why the cue card but not the shape controlled HD cells. Alternatively, perhaps the difference is categorical: cells will always follow a cue card but never follow geometry, regardless of salience. We tested this hypothesis in the next manipulation. To reduce the visual saliency of the cue card and thus make it visually more comparable to the geometry-only conditions, a dark gray cue card was attached to the shortest wall of the isosceles triangle.

\section{Condition 5: Triangle plus gray cue card}

A total of $15 \mathrm{HD}$ cells in seven ensembles were recorded from five rats during seven sessions when a gray A3 cue card was attached to the shortest wall of the triangle. An example of a trial in the gray cue card condition is shown in Figure $4 E$. In this example, the HD cell clearly rotates on two trials but fails to rotate on the other two trials. This combination of HD rota-

tions and fixations were typical of sessions in this condition. As the data points in Figure 5 represent total shifts in each fourrotation session, these intertrial variations have been averaged.

Overall, for all ensembles during a $90^{\circ}$ triangle plus gray card rotation, the mean shift in firing direction was $41^{\circ}$, with a resultant vector length of 0.73 (Fig. $5 E$ ). A Rayleigh test showed that these data significantly clustered around the $41^{\circ} \operatorname{shift}(r=5.08$, $p<0.01$ ) and were not significantly different from zero (upper $\mathrm{CI}=82.51$, lower $\mathrm{CI}=-0.57$ ). Note that the confidence intervals show the values to be just significant. However, we did find a final deviation-from-expected value of $49^{\circ}( \pm 42.46$ $\mathrm{SD}$ ), which was significantly different from zero (upper CI $=$ 91.1, lower CI = 7.45). Together, these results indicate that the rotations of the firing directions of the HD cells were significantly different from rotations of the triangle plus gray card environment.

For all ensembles during a $180^{\circ}$ triangle plus gray card rotation, the mean shift in firing direction was $-143^{\circ}$, with a resultant vector length of 0.11 (Fig. 5E). A Rayleigh test showed that these data did not significantly cluster around the $-143^{\circ}$ value $(r=0.8$, NS), indicating that the cells in this condition showed large variations in the amount of firing direction rotation. distribution is averaged out $\left(\sim 40^{\circ}\right)$.

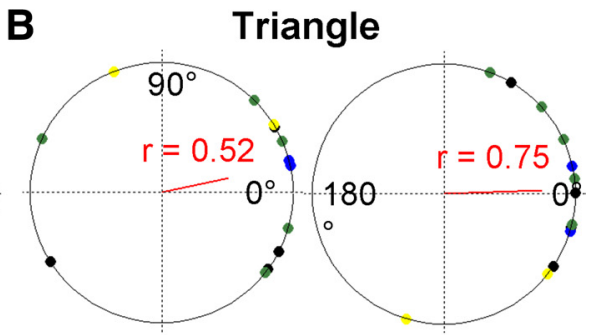

D White Cue Card

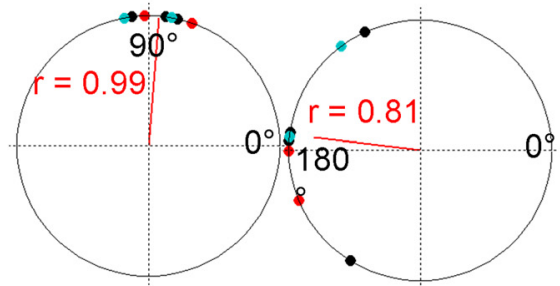

F Disorientation

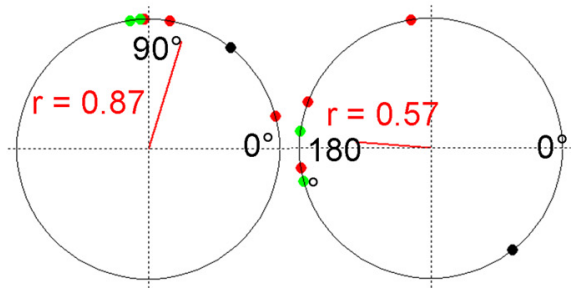

Figure 5. $A-F$, Circular plots showing the mean shift of $H D$ cells in each condition for $90^{\circ}$ rotations (left plots) or $180^{\circ}$ rotations (right plots). Ninety degree rotations were normalized for direction by reflecting the data from clockwise rotations onto counterclockwise rotations. The degree to which the environments were shifted (either $90^{\circ}$ or $180^{\circ}$ ) is indicated on each plot. Each dot 列 列 indicates a greater clustering of data points. Note that in the $90^{\circ}$ rotations for the gray cue card condition $(\boldsymbol{E})$, cells tended to follow the environment during one rotation but not both rotations in the session. As the data points represent one session, this bimodal

The cells during the gray cue card condition therefore failed to rotate with the gray card. However, we did observe that cells tended to rotate with the environment during one rotation, but never for all five rotations within a session. One possible explanation for this is that, unlike a visually salient white cue card, which could arguably be seen by the rats from anywhere in the environment, information about the gray cue card might only be processed if the rat was close to the card, by detection of tactile, olfactory, or weak visual cues. If the rat did not visit the card early on in a trial, this information might be processed after the HD circuit had established a stable orientation, resulting in a failure of the gray card to gain control over the cells. We therefore examined the path of the rats in this gray cue card condition to estimate when they first approached the cue card. The average time that a rat took to first approach the gray cue card when the deviation-from-expected value for the trial was $<20^{\circ}$ was $14.6 \mathrm{~s}$. The average approach time when the deviation-from-expected for the trial was $>20^{\circ}$ was $15.0 \mathrm{~s}$. An independent samples $t$ test showed that these approach times were not significantly different $\left(t_{(33)}=0.055, \mathrm{NS}\right)$. These findings suggest that this proximity hypothesis is unlikely. However, no behavioral measures were taken during the trial, so definitive conclusions cannot be drawn. 
Table 3. Table of statistics for each condition

\begin{tabular}{|c|c|c|c|c|c|c|}
\hline & Trapezoid & Triangle & Teardrop & White cue card & Gray cue card & Disorientation \\
\hline \multicolumn{7}{|l|}{$180^{\circ}$} \\
\hline AMD & $-166^{\circ}$ & $178^{\circ}$ & $168^{\circ}$ & $7^{\circ}$ & $37^{\circ}$ & $5^{\circ}$ \\
\hline \multicolumn{7}{|l|}{ Trapezoid } \\
\hline Triangle & $F_{(1,19)}=0.82$ & & & & & \\
\hline Teardrop & $F_{(1,13)}=3.15$ & $F_{(1,18)}=0.29$ & & & & \\
\hline $\begin{array}{l}\text { White cue card } \\
\text { Gray cue card }\end{array}$ & $F_{(1,14)}=85.23$ & $F_{(1,19)}=58.31$ & $F_{(1,13)}=69.41$ & & & \\
\hline Disorientation & $F_{(1,12)}=6.76$ & $F_{(1,17)}=23.54$ & Sample sizes too small & $F_{(1,12)}=0.01$ & & \\
\hline \multicolumn{7}{|l|}{$90^{\circ}$} \\
\hline AMD & $91^{\circ}$ & $77^{\circ}$ & $67^{\circ}$ & $5^{\circ}$ & $49^{\circ}$ & $17^{\circ}$ \\
\hline \multicolumn{7}{|l|}{ Trapezoid } \\
\hline Triangle & $F_{(1,19)}=0.32$ & & & & & \\
\hline Teardrop & $F_{(1,13)}=1.57$ & $F_{(1,18)}=0.14$ & & & & \\
\hline White cue card & $F_{(1,14)}=61.77$ & $F_{(1,19)}=10.09$ & $F_{(1,13)}=15.27$ & & & \\
\hline Gray cue card & $F_{(1,13)}=4.36$ & $F_{(1,18)}=0.33$ & $F_{(1,11)}=0.54$ & $F_{(1,13)}=6.34$ & & \\
\hline Disorientation & $F_{(1,12)}=19.62$ & $F_{(1,17)}=4.8$ & Sample sizes too small & $F_{(1,12)}=1.02$ & $F_{(1,11)}=1.86$ & \\
\hline
\end{tabular}

Comparisons of absolute mean deviations (AMD) for each condition during $180^{\circ}$ and $90^{\circ}$ rotations of the environment. Significant differences with Bonferroni corrections are in bold.

The failure of cells to rotate with the gray cue card suggests that one possible reason for a lack of HD rotation in the geometry-only conditions is poor visual saliency. It could therefore be argued that HD cells can theoretically use geometric information but were unable to in this situation because the geometric cues had low salience and did not override other information sources. The final condition was designed to test this possibility by disrupting the path integration signal, a manipulation that has been shown to increase the use of geometric cues in behavioral studies (Batty et al., 2009). With fewer sources of competing directional information, geometric cues might now be able to influence HD orientation.

\section{Condition 6: Disorientation}

In this condition, rats were disoriented by the experimenter manually spinning the holding box (approximately one rotation per second) while walking around the curtained enclosure for $\sim 1$ min before recording.

A total of $10 \mathrm{HD}$ cells in four ensembles were recorded from three rats during six disorientation sessions in the triangle. An example of a disorientation trial is shown in Figure $4 F$. Note that, like the white cue card example, in this representative example, the firing direction of the cell reliably followed the triangle.

Overall, for all ensembles during a $90^{\circ}$ disorientation plus triangle rotation, the mean shift in firing direction was $73^{\circ}$, with a resultant vector length of 0.87 (Fig. $5 F$ ). A Rayleigh test showed that these data significantly clustered around the $73^{\circ}$ shift $(r=5.24, p<0.01)$ and were significantly different from zero (upper CI $=107.14$, lower CI $=39.53$ ), indicating that the cells did not remain oriented to the room. Correspondingly, the final deviation-from-expected value of $17^{\circ}( \pm 28.87$ SD) was not significantly different from zero (upper CI = 50.99, lower CI $=-16.62$ ). Together, these results suggest that the rotations of the firing directions of the HD cells were not significantly different from rotations of the triangle.

Similarly, for all ensembles during a $180^{\circ}$ disorientation plus triangle rotation, the mean shift in firing direction was $175^{\circ}$, with a resultant vector length of 0.57 (Fig. $5 F$ ). A Rayleigh test showed that these data significantly clustered around the $175^{\circ}$ value $(r=5.24, p<0.05)$ and were significantly different from zero (upper CI $=107.14$, lower $C I=39.53$ ), indicating that the cells did not lock onto the room cues. Correspondingly, we found a final deviation-from-expected value of $5^{\circ}( \pm 53.12 \mathrm{SD})$, which was not significantly different from zero (upper CI $=50.99$, lower $\mathrm{CI}=-16.62$ ), indicating that the firing directions remained generally aligned to the geometry of the triangle.

This indicates that when the rat was disorientated, the HD cells used the geometric features of the triangle. This consequently suggests that in the basic triangle condition (where no disorientation occurred), geometric cues were potentially available to the HD cells, but were unable to override other uncontrolled directional cues such as path integration.

\section{Comparison between conditions}

It was predicted that because the conditions varied in the degree to which rats needed to process geometry to orient, the influence of geometry in each condition would also vary. More precisely, it was thought that the trapezoid would cause the HD cells to rotate the least, followed by the triangle, the teardrop, the gray cue card, and finally the white cue card. Overall significant differences were found between all the conditions for $180^{\circ}\left(F_{(5,48)}=14.69, p<\right.$ $0.001)$ and $90^{\circ}\left(F_{(5,48)}=4.34, p<0.01\right)$ rotations. Circular $t$ tests were then used to do pairwise comparisons (with Bonferroni corrections) between all the conditions. All comparisons are shown in Table 3 for $180^{\circ}$ and $90^{\circ}$ rotations. Due to the small $r$ value in the $180^{\circ}$ gray cue card condition, the parametric test could not be used. However, sample sizes were too small to use the nonparametric test.

Table 3 show that there were no significant differences between the absolute mean deviations in the trapezoid, triangle, and teardrop conditions, which were the conditions in which firing directions failed to follow the enclosures. Similarly, there were no significant differences between the white cue card condition and the disorientation condition, which were the two conditions in which firing did follow the enclosures. Significant differences were found, however, with all the pairwise comparisons between the geometry-only conditions (trapezoid, triangle, and teardrop) and the white cue card and with the majority of comparisons between the geometry-only conditions and the disorientation condition, with the absolute mean deviations in the white cue card and disorientation conditions being significantly smaller (Fig. 6). 


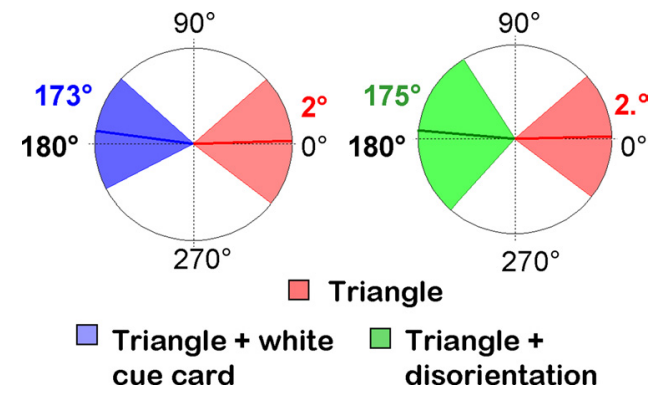

Figure 6. Circular plots showing the mean HD cell shifts (lines) and confidence intervals (shaded areas) during $180^{\circ}$ rotations for the triangle condition (red), the white cue card condition (blue), and the disorientation condition (green). The two graphs show that adding a proximal landmark (left) or disorientating the rat (right) produce significantly greater shifts in HD cells compared with a condition where geometric information was pitted against a stable path integration cue.

\section{Discussion}

This study explored the use of geometric cues by HD cells to determine whether geometry is an important source of orienting information (Cheng, 1986). We found that when rats were not disoriented, HD cells did not use even a highly polarized environmental geometry to orient their firing directions. Instead, firing directions generally remained aligned to the room. When rats were strongly disoriented, HD cells rotated with the bare enclosures. This demonstrates that HD cells can process geometry when other cues, such as landmarks and path integration, are unavailable, but this effect was relatively weak and failed to override path integration. This finding is important in light of previous suggestions that geometric cues are preferentially involved in reorientation-the so-called "geometric module hypothesis" (Cheng, 1986, 2008). Our results suggest this may be the case only when path integration is severely disrupted following disorientation.

There are two aspects of note in our findings: first, that HD cells did not appear sensitive to geometry in oriented rats; and second, that they were sensitive in disoriented rats. These two situations are discussed in turn below.

\section{Influence of geometric cues in oriented animals}

Relatively few behavioral studies have examined the influence of geometry in nondisoriented animals. Lourenco and Huttenlocher (2006) found that children used geometric cues to find a goal when disoriented by self-rotation, but did not use the same cues when it was the environment that had been rotated instead. Batty et al. (2009) found that disorientation of rats during training caused a relative preference for the use of geometric cues (as opposed to internal, path integration cues) during testing, although disorientation before the test itself did not increase the use of geometry any further. It appears from these studies that when orientation (path integration) cues were present, they influenced choices: findings that are consistent with our observation that when orientation or visual cues were present, HD cells apparently preferred them over geometry.

Studies of neuronal responses to changes in geometry have, by contrast, produced results that seem to contradict ours. Kubie and Ranck (1983) and Muller and Kubie (1987) found that hippocampal place cells remapped when nondisoriented animals were placed in environments of differing geometry. However, the environments also differed in nongeometric aspects (e.g., the material of the walls), information to which place cells are known to be sensitive (Anderson and Jeffery, 2003). It is not known whether HD cells changed orientation following these manipulations. These studies therefore do not speak directly to the issue of HD cell geometric processing.

Direct observation of HD cells has also, however, suggested a possible influence of geometry. Golob et al. (2001) found that $\mathrm{HD}$ cells changed orientation in response to the shift of the animals (without disorientation) from a square enclosure to a rectangular one. In these experiments, rats were trained to locate a corner of the square arena, which was polarized with a cue card, for water reward. When the arena was changed to a rectangle, cells rotated their firing directions on 12 of 13 occasions. These results suggest the processing of geometric cues by HD cells; however, most of the shifts in firing direction were multiples of $90^{\circ}$, suggesting that what changed was the cells' choice of corner to orient by. Given that the rats had been trained to specifically attend to corners to locate the reward, it is possible the HD cells were using corners as landmarks, rather than processing geometry as a holistic property of the environment. Thus, it is not clear whether this alteration represents true geometric processing by the HD cells.

An apparent effect of environment geometry on HD cells was also found by Dudchenko and Zinyuk (2005), who recorded HD cells in two T-mazes that were located in adjacent rooms and oriented differently. They found that HD cells rotated between the two rooms so as to maintain the same orientation with respect to the maze. However, while the cells were clearly orienting using the apparatus, it may not have been geometry per se that the cells were using. The rats were always started from the same place in the maze, and studies of place cells have shown that start position can be an orienting cue for place cells (Sharp et al., 1990), presumably via the head direction cells. Furthermore, the maze would provide a visually unique panorama from most locations, which means the HD cells would have potential visual orienting cues even if they did not process geometry directly.

Thus, although prior studies of HD cells have shown responsiveness to manipulations of environmental geometry, it has not been shown that the relevant factor was geometry itself and not some feature or other cue also present. The fact that our environments were relatively low in unique features, despite their distinctly polarized geometry, may explain why HD cells in our oriented rats preferred to use path integration or the cue card when these were available.

\section{Influence of geometric cues in disoriented animals}

Our finding that geometric cues by themselves have only a weak influence on HD cell orientation seems surprising given the substantial behavioral literature suggesting a primacy for geometric cues over landmarks (Cheng, 1986; for review, see Cheng and Newcombe, 2005). However, most behavioral studies have been undertaken in disoriented rats (Cheng, 1986; Maurer and Derivaz, 2000; Wall et al., 2004; Cheng and Gallistel, 2005; Skov-Rackette and Shettleworth, 2005; Gibson et al., 2007; Maes et al., 2009), in which path integration has been disrupted. The present results suggest that geometric cues are able to influence HD cells if other sources of orienting information are absent.

When other orienting cues are absent, our findings accord with previous research on HD cells. Taube et al. (1990b) found that when a square enclosure was changed to a rectangle, HD cells in disoriented rats rotated their firing directions, while Golob and Taube (1997) found that HD cells in disoriented 
rats with hippocampal lesions altered their firing when an enclosure was changed from a cylinder to a square, triangle, or pentagon. More recently, Clark et al. (2010) found that geometric cues could override the effect of a single landmark. These rotations may have been due to geometric processing, but may, as noted above, also result from processing of specific features arising from the geometry. Further experiments will be needed to test whether true geometric processing occurs during reorientation.

Interestingly, Clark et al. (2010) found that geometric cues tended not to override the effects of multiple landmarks, suggesting that the hierarchy of cue preference is salience-dependent rather than absolute. We found the use of a landmark to be salience-dependent when we manipulated its visual contrast. Thus, the switch between the use of different cues may result from salience-based competition rather than any absolute hierarchy of cue use. Previous studies have also found a switch in directional cue use by HD cells under some circumstances. For example, Knierim et al. (1995) found that a cue card was more likely to gain control over HD cells in rats that had learned about the cue while they were nondisoriented than in rats that had been disoriented during training. All animals were tested under the same disorientation conditions, suggesting that the cue card had lost salience because of the disorientation-training procedure. This finding is reminiscent of the Batty et al. (2009) behavioral study, in which disorientation during training caused a reduction in the use of geometric cues during testing. These experiments show that the influence of directional cues on HD cells can be reweighted with experience and is not absolute.

Our results additionally indicate that the orientation state of the animal at the time of testing influences cue use too. The finding that disorientation instantaneously modulates the relative influence of geometric cues is interesting because disorientation does not degrade the HD signal. This suggests that whatever decision process is responsible for assigning precedence to an input, it knows about the state of the path integrator. Thus, it seems able to distinguish between mild disorientation, when there is merely a slight disagreement between the path integrator and the environmental cues (as when the shape was rotated), and complete disorientation. HD cells seemed to use path integration in the former case and geometry in the latter. Since HD cells in thoroughly disoriented rats still fired strongly and with tight (albeit misdirected) tuning curves, the implication is that the signal about orientation state (mild vs severe), and thus the decision to use or ignore geometry, occurs outside the HD cells themselves. Potentially, there is an input that tells the HD cells that severe disorientation has occurred and therefore to weight external cues, including geometry, more heavily than normal. Such a scheme could provide an interesting model system for the study of Bayesian integration processes (Cheng et al., 2007).

Why, adaptively speaking, should geometry be such a weak cue for HD cell orientation? We can only speculate, but it is worth noting that the HD cell system is probably old, in evolutionary terms, whereas the ecological lifestyles of rats living in bounded spaces are more recent. Many animals that share a common ancestor with rodents, such as fish and birds, live in open spaces that lack a clear geometry. Furthermore, it is not clear that even in bounded spaces, it is geometry per se that is the relevant feature, rather than the visual panorama that derives from geometric configurations (Cheung et al., 2008; Stürzl et al., 2008). Further work is needed to determine what aspect of geometry influences HD cells in disoriented rats.

In summary, we have explored the use of geometric cues by HD cells and found that, in nondisoriented rats, their influence does not appear as strong as the influence of other simultaneously present cues such as a landmark or path integration. Conversely, in disoriented rats, in which path integration was disabled, geometry did have an effect, suggesting that it is processed, even if its effect is only weak. Since disorientation is presumably an unusual condition in the normal life of a rat, the implication is that local geometry is not a primary guide to the HD system in rodents, but may be used as a weak backup system for reorientation when other cues are unavailable.

\section{References}

Anderson MI, Jeffery KJ (2003) Heterogeneous modulation of place cell firing by changes in context. J Neurosci 23:8827-8835.

Batty ER, Hoban L, Spetch ML, Dickson CT (2009) Rats' use of geometric, featural and orientation cues to locate a hidden goal. Behav Processes $82: 327-334$.

Berens P (2009) CircStat: a MATLAB toolbox for circular statistics. J Stat Softw 31:1-21.

Cheng K (1986) A purely geometric module in the rat's spatial representation. Cognition 23:149-178.

Cheng K (2008) Whither geometry? Troubles of the geometric module. Trends Cogn Sci 12:355-361.

Cheng K, Gallistel CR (2005) Shape parameters explain data from spatial transformations: comment on Pearce et al (2004) and Tommasi and Polli (2004). J Exp Psychol Anim Behav Process 31:254-259.

Cheng K, Newcombe NS (2005) Is there a geometric module for spatial orientation? Squaring theory and evidence. Psychon Bull Rev 12:1-23.

Cheng K, Shettleworth SJ, Huttenlocher J, Rieser JJ (2007) Bayesian integration of spatial information. Psychol Bull 133:625-637.

Cheung A, Stüzl W, Zeil J, Cheng K (2008) The information content of panoramic images. II. View-based navigation in nonrectangular experimental arenas. J Exp Psychol Anim Behav Process 34:15-30.

Clark BJ, Harris MJ, Taube JS (2010) Control of anterodorsal thalamic head direction cells by environmental boundaries: comparison with conflicting distal landmarks. Hippocampus. Advance online publication. Retrieved Nov 25, 2010. doi:10.1002/hipo.20880.

Dudchenko PA, Goodridge JP, Taube JS (1997) The effects of disorientation on visual landmark control of head direction cell orientation. Exp Brain Res 115:375-380.

Gibson BM, Wilks TJ, Kelly DM (2007) Rats (Rattus norvegicus) encode the shape of an array of discrete objects. J Comp Psychol 121:130-144.

Golob EJ, Taube JS (1997) Head direction cells and episodic spatial information in rats without a hippocampus. Proc Natl Acad Sci U S A 94: 7645-7650.

Golob EJ, Stackman RW, Wong AC, Taube JS (2001) On the behavioral significance of head direction cells: neural and behavioral dynamics during spatial memory tasks. Behav Neurosci 115:285-304.

Knierim JJ, Kudrimoti HS, McNaughton BL (1995) Place cells, head direction cells, and the learning of landmark stability. J Neurosci 15:1648-1659.

Kubie, J and Ranck J-B Jr (1983) Sensory-behavioral correlates in individual hippocampus neurons in three situations: space and context. In: The hippocampus (Seifert W, ed), pp 433-447. New York: Academic.

Leutgeb S, Ragozzino KE, Mizumori SJ (2000) Convergence of head direction and place information in the CA1 region of hippocampus. Neuroscience 100:11-19.

Lourenco SF, Huttenlocher J (2006) How do young children determine location? Evidence from disorientation tasks. Cognition 100:511-529.

Maes JH, Fontanari L, Regolin L (2009) Spatial reorientation in rats (Rattus norvegicus): use of geometric and featural information as a function of arena size and feature location. Behav Brain Res 201:285-291.

Maurer R, Derivaz V (2000) Rats in a transparent Morris water maze use elemental and configural geometry of landmarks as well as distance to the pool wall. Spatial Cogn Comput 2:135-156.

Muller RU, Kubie JL (1987) The effects of changes in the environment on the spatial firing of hippocampal complex-spike cells. J Neurosci 7:1951-1968.

Paxinos G, Watson C (2004) The rat brain in stereotaxic coordinates. New York: Academic. 
Pecchia T, Vallortigara G (2010) Reorienting strategies in a rectangular array of landmarks by domestic chicks (Gallus gallus). J Comp Psychol 124:147-158.

Sharp PE, Kubie JL, Muller RU (1990) Firing properties of hippocampal neurons in a visually symmetrical environment: contributions of multiple sensory cues and mnemonic processes. J Neurosci 10:3093-3105.

Skov-Rackette SI, Shettleworth SJ (2005) What do rats learn about the geometry of object arrays? Tests with exploratory behavior. J Exp Psychol Anim Behav Process 31:142-154.

Stürzl W, Cheung A, Cheng K, Zeil J (2008) The information content of panoramic images. I. The rotational errors and the similarity of views in rectangular experimental arenas. J Exp Psychol Anim Behav Process 34:1-14.

Taube JS (1995) Place cells recorded in the parasubiculum of freely moving rats. Hippocampus 5:569-583.
Taube JS, Muller RU, Ranck JB Jr (1990a) Head-direction cells recorded from the postsubiculum in freely moving rats. I. Description and quantitative analysis. J Neurosci 10:420-435.

Taube JS, Muller RU, Ranck JB Jr (1990b) Head-direction cells recorded from the postsubiculum in freely moving rats. II. Effects of environmental manipulations. J Neurosci 10:436-447.

Taube JS, Goodridge JP, Golob EJ, Dudchenko PA, Stackman RW (1996) Processing the head direction cell signal: a review and commentary. Brain Res Bull 40:477-484.

van der Meer MA, Richmond Z, Braga RM, Wood ER, Dudchenko PA (2010) Evidence for the use of an internal sense of direction in homing. Behav Neurosci 124:164-169.

Wall PL, Botly LC, Black CK, Shettleworth SJ (2004) The geometric module in the rat: independence of shape and feature learning in a food finding task. Learn Behav 32:289-298. 